\title{
The epidemiology and genomics of a virulent emerging fungal pathogen in an
}

\section{Australian reptile}

\author{
B. Class ${ }^{1}$, D. Powell ${ }^{1}$, J. Terraube ${ }^{1}$, G. Albery ${ }^{2}$, C. Delmé ${ }^{1}$, S. Bansal ${ }^{2}$, C.H. Frère ${ }^{1,3^{*}}$
}

${ }^{1}$ Global Change Ecology Research Group, University of the Sunshine Coast; Sippy Downs, QLD, Australia.

${ }^{2}$ Department of Biology, Georgetown University; Washington DC, United States.

${ }^{3}$ School of Biological Sciences, University of Queensland, St Lucia, QLD, Australia

*Corresponding author. Email: c.frere@uq.edu.au

\begin{abstract}
Emerging infectious fungal diseases (EIFDs) represent a major conservation concern worldwide. Here, we provide early insights into the potential threat that Nannizziopsis barbatae $(\mathrm{Nb})$, a novel EIFD, poses to Australian herpetological biodiversity. First known to the reptile pet trade as a primary pathogen causing untreatable severe dermatomycosis, since $2013, N b$ has emerged in a growing number of phylogenetically and ecologically distant free-living reptiles across Australia. Observing its emergence in a long-term study population of wild eastern water dragons (Intellagama lesueurii), we demonstrate the pathogen's virulence-related genomic features, within-population spatiotemporal spread, and survival costs, all of which imply that $N b$ could pose a threat to Australian reptiles in the future. Our findings highlight the need to closely monitor this pathogen in Australian ecosystems.
\end{abstract}




\section{Introduction}

2 Emerging infectious fungal diseases (EIFDs) pose a serious threat to the conservation of global biodiversity (1-3) and are responsible for some of the most severe mass mortality events in wild populations (1-4). Notable examples include chytridiomycosis, which has now impacted over 500 species of amphibians in 54 countries, driving the extinction of 90 species worldwide (5); whitenose syndrome, which has resulted in a devastating $75 \%$ population decline across bats in Canada and the USA $(6,7)$; and the more recent snake fungal disease $(8)$, which poses a significant threat to snake populations in eastern North America (9). Whilst EIFDs make up less than three percent of infectious agents reported amongst animal hosts, they are nonetheless responsible for over 70 $\%$ of disease-driven population declines and extinctions (1).

11 Members of the fungal genus Nannizziopsis are well known to the pet trade as primary pathogens that cause serious cutaneous and systemic fatal disease in a diverse range of reptiles across the world (10-13). Nannizziopsis barbatae was first identified in captivity in 2009 (14), and remained confined to captivity until, in 2013, two-free living eastern water dragons (Intellagama lesueurii) from locations separated by $30 \mathrm{~km}$ across Brisbane (Queensland, Australia) were identified with

16 proliferative dermatitis, necrosis, ulceration and emaciation (15). $\mathrm{Nb}$ has since emerged in a 17 growing number of phylogenetically and ecologically distant free-living lizards $(2 \times$ agamid species and 2 x skink species) across Australia (6 sites in Qld, 1 site in NSW and 1 site in WA)

19 (15) and is known to cause disease in 9 species (data combined from captive and wild cases, see Table S1). This recent emergence in the wild, followed by a rapid expansion of its geographical

21 distribution and host range, indicate that this fungal pathogen may present a pressing new threat to Australia's herpetological biodiversity. While we know that $\mathrm{Nb}$ causes untreatable severe dermatomycosis (15), mitigating its impact will require a thorough understanding of its ecology. 
24 Taking advantage of its recent emergence in a long-term study population of eastern water dragons

25 (15) and using an innovative combination of comparative genomics and spatiotemporal

26 autocorrelation models, we assess the potential threat that $N b$ may pose to Australia's

27 herpetological biodiversity.

\section{Results}

$N b$ emerged in 2013 in two geographically isolated populations of eastern water dragons in the city of Brisbane (QLD, Australia). One of these populations (Roma Street Parkland, 27² $27^{\prime} 46^{\prime} \mathrm{S}$, $153^{\circ} 1^{\prime} 11^{\prime} \mathrm{E}$ ) has been monitored with frequent behavioral surveys and yearly catching since 2010.

This population comprises 336 individuals on average and behavioral surveys were performed 23 times per week along a transect which covered $85 \%$ of this population (16). During behavioral surveys, individuals' GPS position were systematically recorded and profile photographs taken to allow later identification based on unique scale patterns (17). Disease diagnosis was based on the presence or absence of characteristic skin lesions (15), which observers were trained to recognize from season 9 (2018-2019) onwards. Individuals' disease status for earlier years was hence determined retrospectively using photos from catching and, when not available, behavioral surveys. Once diagnosed, individuals were assumed to remain diseased even when not caught again. Amongst the diseased individuals repeatedly captured between February 2020 and August 2021, some individuals (20/61) showed a reduction in the severity of their lesions, although this reduction was mainly observed in individuals exhibiting mild lesions (15/20, Data S1). Using this decade-long individual-based data, we found that the disease prevalence has continuously increased throughout the population since $N b$ 's emergence. Starting with one individual in 2013, a total of 158 individuals have now presented with clinical signs of the disease $(n=1221$ for field seasons 3-11) and in the last field season (2020-2021), the prevalence was $26.4 \%$ (95\%CI: 24.228.4, Fig. S1, Table S2). The majority (96.7\%) of these individuals were adults, and males 
(58.3\%). Only five juveniles were found with clinical signs of the disease $(0.07$ to $5.5 \%$ of juveniles) between late 2018 to early 2021, despite juveniles representing on average $17.8 \%$ of observed individuals during these years.

\section{N. barbatae shares genomic characteristics with other fungal pathogens}

$\mathrm{Nb}$ is a member of the Onygenales, an order of fungi that are able to degrade keratin, the main component of the vertebrate outer skin layer. Some members of this order are important primary pathogens of animals and humans and recent comparative genomic studies have helped resolve differences in gene content between pathogenic and non-pathogenic species (18). With this in mind, we performed a comparative whole-genome analysis incorporating the full set of genes of $N b$ (8,012 predicted protein-coding sequences) together with 16 other species of fungi (Table 1) to uncover genomic features likely to contribute to $\mathrm{Nb}$ 's pathogenicity.

First, we identified that the $N b$ genome contains a gene repertoire rich in proteases, known to increase fungal virulence (19), and shares similarities with other pathogenic Onygenaceae (Fig. 1A). Most notable is an expansion of trypsin domain-containing genes (PF00089) (Fig. 1B) found only in $\mathrm{Nb}$ (7 genes) and the fungus causing snake fungal disease, O. ophidiicola (29 genes). Both of these species are capable of primary infection in reptiles $(9,20)$ suggesting a role for this gene family in influencing host range. $N b$ has a degradome that bears resemblance to important dermatophytes and the enrichment of proteases including, subtilase (PF00082) and deuterolysin (PF02102), suggest extensive proteolytic capacity. Second, $\mathrm{Nb}$ has a large number of protein kinase domain-containing genes (PF00069) which may contribute to its capacity to infect a broad range of reptile taxa (15). Last, we identified a higher number of LysM domain-containing genes (PF01476) in the $N b$ genome than most of the other fungi in this analysis. Together, these 
characteristics of the $\mathrm{Nb}$ genome highlight factors which may be key to determining its propensity to infect herpetofauna.

\section{N. barbatae infection is spatially structured within the population}

To investigate the phenotypic and spatiotemporal predictors of fungal infection, we constructed spatiotemporal autocorrelation models using the Integrated Nested Laplace Algorithm. Comparison of disease prevalence models (Fig. S2) provided strong support for spatial structuring $(\triangle \mathrm{DIC}=-117.24$ relative to the base model), but relatively little evidence for spatiotemporal structuring $(\triangle \mathrm{DIC}=-4.11$ relative to the spatial model) $($ Fig. $\mathrm{S} 2)$. That is, disease prevalence varied more spatially (assuming no time effect) than spatiotemporally. Indeed, spatial effects were strongly correlated across field seasons (rho>0.9) and disease prevalence has remained consistently higher in the East (up to $33 \%$ ) compared to the West (<10\%, Fig. 2). Models also showed lower prevalence in juveniles than adults, and in females compared to males (Fig. S2).

\section{N. barbatae infection is associated with survival costs}

To investigate the survival costs of infection, we fitted a binomial survival model, where survival of an individual was coded based on whether they were observed in any subsequent year. The full population model showed effects of cohort, field season and sex on the yearly probability of survival but failed to detect any effect of the disease (Fig. 3A). In contrast, randomly subsampling diseased and non-diseased individuals from matching cohorts and accounting for age (number of days in the population), sex, and field season in subsequent models revealed a small but significant individual survival costs of the disease (Fig. 3B-D). All subsampled models found a significant effect of the disease (Fig. 3B); the overall mean survival cost was $12 \%$ (Fig. 3C), so that the mean predicted annual survival of diseased individuals was $74 \%$ compared to $86 \%$ for non-diseased 
individuals (Fig. 3D). Controlling for spatial autocorrelation did not improve model fit ( $\triangle \mathrm{DIC}>-2$ relative to the base model), demonstrating that this survival cost did not vary spatially.

\section{Discussion}

Gaining early insights into disease virulence, spatiotemporal spread, and survival costs is particularly urgent in the case of novel emerging infections that have the potential to severely threaten biodiversity. Yet such data are challenging to obtain in the wild, greatly impeding our abilities to predict and mitigate the impact of infections on wildlife. Our study investigates withinpopulation spread and impacts of Nannizziopsis barbatae, a novel emerging infectious fungal disease which should give us cause for vigilance.

\section{Genomic signatures of pathogenicity}

We show that the free-living $N b$ genome sampled from our long-term study population of eastern water dragon contains many gene families implicated in fungal pathogenicity, including several proteases, protein kinases, and LysM effector proteins. Virulence in wildlife fungal pathogens has often been associated with expansions of protease gene repertoires and their expression (e.g. chytrid (21); WNS (22-24)). Nb has a gene repertoire rich in proteases with features similar to those identified in other wildlife fungal pathogens such as snake fungal disease (e.g. trypsin domain-containing genes) and chytrid fungus (e.g. M36 metalloproteases). Additionally, a comprehensive and novel repertoire of protein kinases can provide fungal species with plasticity in occupying different ecological niches and responding to environmental change $(25,26)$. LysM effector proteins may contribute to fungal virulence by suppressing the host immune system response via interactions with chitin (27). Comparative studies strongly suggest an association with the enrichment of LysM domain-containing genes and virulence in the keratin-degrading dermatophytes (26). Furthermore, chitin-binding CBM18 gene family proteins (PF00187) are also 
115 found expanded in $B$. dendrobatidis, and are thought to play a role in evasion of the amphibian

116 host immune response (26). While understanding the molecular mechanisms of this pathogen is

117 central to mitigating its impact on wildlife, the exact source of $\mathrm{Nb}$, its current free-living genetic

118 diversity, and its mode of introduction into our dragon population remain unknown. Genomic

119 resources for Nannizziopsis spp. will enable the development of tools to answer these questions.

120 The emergence of this disease urgently necessitates the identification of its origin to better

121 understand and thus predict the impact it will likely have on the Australian herpetofauna. For

122 instance, it is critical that we determine whether or not we are dealing with a novel pathogen and

123 thus naïve hosts, or whether the population has had historical exposure to the pathogen.

\section{Within-population spread}

125 Even though central to the forecasting and control of wildlife disease management, quantifying

126 the contribution of different transmission pathways of a pathogen is notoriously challenging to

127 achieve in nature (28). Using an intensively-studied lizard population, we provide a much-needed

128 early assessment of $\mathrm{Nb}$ 's spatiotemporal spread since its emergence in 2013 . We show that the

129 disease has spread relatively rapidly across an increasing portion of the population, providing the

130 first likely evidence for within-population $\mathrm{Nb}$ transmission in the wild. From a single individual

131 dragon identified with $N b$-like clinical signs in 2013, more than 150 individuals have displayed

132 apparent clinical signs of $N b$ and the disease prevalence has reached $26.4 \%$ of the population.

133 Worryingly, prevalence of the disease has been continuously increasing since 2016 and shows no

134 signs of slowing down. Although it is unclear what the transmission route of this pathogen is in

135 this population (e.g. physical contact or environmental latency), our results show that over the

136 years, the disease prevalence has remained higher in the eastern part of the park than in the western

137 area, which could be due to spatial variation in environmental factors influencing the pathogen's 
survival, transmission, or virulence (29). Analyses of the dragons' spatial and social behaviors coupled with molecular diagnostics capabilities will help identify transmission routes, predict geographic spread of the pathogen, and inform potential future interventions (30).

\section{Low but detectable survival costs}

Survival costs of $\mathrm{Nb}$ infection were detectable at an individual level. Although individuals showing clinical signs of the disease varied in their survival costs, they were still relatively likely to survive from one year to the next (>70\% chance), demonstrating that adults are relatively tolerant to the pathogen and can carry it for multiple years once skin lesions become apparent. Although the disease has been shown to be incurable in captive reptiles (15), some rare individuals in this population showed reductions in the severity of their lesions (Data S1) and we are yet to determine whether individual diseased dragons can entirely clear the infection (as shown in chytridiomycosis (31) and white-nose syndrome (32)). Additionally, we were only able to detect survival costs when we subsampled our dataset to cohort-matched (age and sex) diseased and non-diseased individuals, thereby reducing extraneous variation in survival probability. Evidence for individual survival costs remains similarly equivocal for other EIFDs, some of which have been studied for much longer than $\mathrm{Nb}$ (31-33). We also acknowledge some uncertainty in our estimates of survival costs due to potential errors in diagnosis, our visual assessment being particularly prone to miss asymptomatic or cryptic infections in the population. Additionally, because lesions are easier to observe in caught individuals, this underestimation may be particularly severe for individuals or classes of individuals that were less likely to be caught (e.g. juveniles). Taken together, these facts imply a general difficulty detecting survival costs of fungal pathogens in long-lived reptiles.

Despite identifying individual-level costs of infection, predicting $\mathrm{Nb}$ 's impacts on population dynamics remains difficult. Such uncertainty is likewise common to other EIFDs, as some 
161 populations affected by chytridiomycosis and white nose syndrome have not declined

162 systematically $(44,45)$. Predicting $\mathrm{Nb}$ 's impacts on the viability of our studied population of

163 eastern water dragons will require key information about: i) $\mathrm{Nb}$ 's prevalence and survival costs at

164 different life stages $(36,37)$, which should be achieved with higher certainty through the use of

165 molecular diagnosis; ii) $\mathrm{Nb}$ 's potential reproduction costs, as was documented for snake fungal

166 disease (38) and chytridiomycosis (39); iii) the mechanisms underlying $N b$ 's spatiotemporal

167 spread. In addition, assessing whether the pathogen's transmission dynamics are density-

168 dependent might be crucial to understanding whether the epidemic will become self-limiting (40).

169 A novel threat for the Australian biodiversity?

170 EIFDs constitute an increasing cause for concern regarding global health, food security and

171 biodiversity conservation (1). With $\mathrm{Nb}$, we may be witnessing the early days of a novel fungal

172 threat to Australian herpetological biodiversity. While other infamous EIFDs with global impacts

173 on wild animal populations were only reported after mass mortality events had already occurred

$174(41,42)$, we have the unique opportunity to monitor the emergence of this pathogen and take action

175 early enough to limit its spread. Although the origin and long-term population impacts of $\mathrm{Nb}$

176 remain unknown, its genomic similarity with other pathogenic EIFDs, capacity to spread in the

177 wild, and detectable survivals costs, combined with its repeated emergence across the country and

178 broad host range, highlight the critical need to closely monitor this pathogen in Australian

179 ecosystems. 
181

182

183

184

185

186

187

188

189

190

191

192

193

194

195

196

197

198

199

200

201

\section{Materials and Methods}

Study system

The population of Eastern water dragons has been monitored since 2010, with frequent behavioral surveys and regular catching during the active season, (i.e. early September to late April). During behavioral surveys (2-3 times per week), individuals' behavior and GPS position were recorded and photographs taken to allow later identification based on unique scale patterns (see (17)). Individuals were also caught during 1-2 weeks catching events in the years 2013, 2014, and yearly since 2016. Morphometric measurements, head and body photographs and DNA samples (blood or tip of the tail) were taken, and unique PIT-tags were inserted in their right upper hind leg. EWD are sexually dimorphic, males being overall larger than females, with more developed jaw and dorsal crest and a red ventral coloration (43). Age class (adult vs. juvenile) was determined for each breeding season using a combination of approaches (snout-vent length when individuals were caught; general appearance when individuals were not caught) and taking into account individuals' observation history (individuals being considered adults after 3 years (43)). Disease diagnosis was based on the presence or absence of characteristic skin lesions (15), which observers were trained to recognize from season 9 (2018-2019) onwards. Individuals' disease status before season 9 was hence determined retrospectively using pictures from behavioral surveys and catching (75-100\% for the latter). From season 9 onwards, disease status was assessed directly in the field during behavioral surveys and catching (65-92\% for the latter). From February 2020 onwards, disease severity was rated for captured individuals using scores ranging from 0 (no lesions, not diseased) to 5 (severely diseased, Table S3). 


\section{Genome annotation and comparative analysis}

The genomes of $N$. barbatae, O. ophidiicola and C. queenslandicum were annotated using the Funannotate (v1.7.4) gene prediction pipeline (https://funannotate.readthedocs.io/). Genomes were firstly screened for repeats using custom generated databases for each species using RepeatModeler (v2.0.1) and masked using RepeatMasker (v4.1.0; http://www.repeatmasker.org). Repeat masked assemblies were then cleaned and sorted before initial gene prediction using GeneMark-ES (v4.65) (44). Protein sequences from high-quality fungal genomes used in this study were used for protein-to-genome alignments as evidence for gene predictors AUGUSTUS (45), SNAP(46), and Glimmer (47) before being passed to EVM (48) to build consensus gene structures. All other predicted protein sequences were downloaded directly from GenBank (Table 1). The newly annotated gene models were evaluated for completeness using BUSCO (v5) (49) in protein mode against the ascomycota_odb10 database (Table S4).

Gene families within each fungal genome were identified from searches of the protein-coding sequences for Pfam (50) domains to assign gene function. We used HMMER (v3.1) (51) (hmmscan) to search the Pfam A database (release 32.0) for 4312 different domains of 16 different species of fungi. To test for significantly expanded gene families, a Fisher's exact test was then conducted iteratively using R (52), comparing the number of counts in Pfam families found in an individual genome, normalised by the total gene count for that species, against the background, which we defined as the average of the counts in the remaining species. Multiple testing corrections were done using the FDR method in $\mathrm{R}$ for all calculated $p$-values. A Pfam domain was considered expanded if it showed a corrected $p$-value $<0.05$. Counts of each domain were collated for each species with domains that occurred multiple times in a protein sequence being counted only once. Heatmap was generated using the package pheatmap with data normalised using the scale function in R. Protein sequences were aligned using Muscle (v3.8.425) (53) and phylogenetic inference 
made using FastTree (v2.1.12) (54) built in to the commercially available Geneious Prime (v2021.1.1) software.

\section{Drivers and spatiotemporal dynamics of infection}

To investigate the phenotypic and spatiotemporal predictors of fungal infection, we constructed spatiotemporal autocorrelation models using the Integrated Nested Laplace Algorithm, implemented in the inla' package in $\mathrm{R}$ (55). These models fitted binary fungal infection as a response variable, where an individual was coded as a 1 if it had previously been diagnosed with fungal infection, and a 0 otherwise. All covariates were categorical, and included Age class (3 levels: Adult, Juvenile, and Unknown); Sex (2 levels: Female and Male); Field season (9 levels: one for each sampling year 2012-2021). The model used a binomial logit error distribution:

$$
\text { Fungus }(0 / 1) \sim \text { Season }+ \text { Sex }+ \text { Age }
$$

We first fitted these fixed effects as a "Base" model. To investigate spatiotemporal patterns of infection, we then added Stochastic Partial Differentiation Equation (SPDE) random effects using individuals' mean map locations in a given season ("annual centroids"). This random effect models two-dimensional patterns of the response variable based on distances between individuals using Matérn correlation $(56,57)$ The "Spatial" model used a static field, where the spatial distribution of infection was modelled to be unchanging across the study period; the "Spatiotemporal" model allowed this field to change from year to year, using an autoregression (AR1) correlation across years, to examine how the infection's distribution changed over the course of the study period. We compared these three models using deviance information criterion (DIC) as a measure of model fit to investigate whether spatiotemporal correlation significantly improved the model. 


\section{Survival costs}

To investigate the survival costs of infection, we fitted a binomial survival model, where survival was coded based on whether the individual was seen in a subsequent year (we hence excluded the most recent year, 2019). The model was specified as follows:

$$
\text { Survived }(0 / 1) \sim \text { Season }+ \text { Sex }+ \text { Cohort }+ \text { ActiveFungus }
$$

Following this model, we used a subsampling routine that allowed us to reduce extraneous variation in survival by compensating for the low proportion of infected individuals in the study period $(120 / 1151=10.4 \%)$ and for the unknown age of infected individuals. We 1) assigned each individual a cohort based on the first season that they were observed in the population; 2) selected the 101 individuals that were ever observed with an infection between 2012 and 2018; and 3) age matched each diseased individual with a random non-diseased individual from their cohort. Between 2018 and 2020, six diseased individuals that were caught in a very poor condition were euthanized. These individuals were hence excluded from these analyses. Having subsampled the population, we then ran the same model as before. This protocol was repeated 1000 times to ensure an even and different selection of non-diseased individuals and survival effect estimates.

We summarized the findings from these models by predicting survival probability for each individual and comparing these values between uninfected and infected individuals. To produce conservative estimates, we randomly drew one effect estimate from each model's fungal effect estimate posterior distribution and used these estimates to predict the survival probability for all infected and uninfected individuals. We then took the mean survival probability for these groups of individuals and subtracted the infected individuals' survival probability from those of the uninfected individuals to estimate a survival cost of infection. 
272 Acknowledgments: We acknowledge the Turrbal and Yugara people, as the First Nations owners

273 of the lands where our study site sits. We pay respect to their Elders, lores, customs and creation

274 spirits. In addition, we would like to thank the students and volunteers that have contributed to the

275 data collection as well as the staff and management of Roma Street Parkland for their ongoing

276 support.

277 Funding:

278 Australian Research Council, grant FT200100192 (CF)

279 Author contributions:

280 Conceptualization: BC, JT, CF, DP, GA, SB

281 Data curation: BC, CD, CF

282 Methodology: GA, DP, BC

283 Investigation: BC, DP, JT, CF, CD

284 Visualization: DP, GA

285 Funding acquisition: $\mathrm{CF}$

286 Project administration: $\mathrm{CF}$

287 Supervision: CF, SB

288 Writing - original draft: BC, JT, CF

289 Writing - review \& editing: BC, JT, CF, DP, GA, SB, CD

290 Competing interests: Authors declare that they have no competing interests. 
291 Data and materials availability: Genome annotation data, individual data and R code used for

292 statistical analyses are available from Figshare using the following link

293 https://doi.org/10.6084/m9.figshare.16599245.

\section{Supplementary Materials}

295 Figs. S1 to S2

296 Table S1 to S4

297 Data S1 


\section{References}

1. M. C. Fisher, Daniel. A. Henk, C. J. Briggs, J. S. Brownstein, L. C. Madoff, S. L. McCraw, S. J. Gurr, Emerging fungal threats to animal, plant and ecosystem health. Nature. 484, 186-194 (2012).

2. M. C. Fisher, N. A. R. Gow, S. J. Gurr, Tackling emerging fungal threats to animal health, food security and ecosystem resilience. Phil. Trans. R. Soc. B. 371, 20160332 (2016).

3. F. Almeida, M. L. Rodrigues, C. Coelho, The still underestimated problem of fungal diseases worldwide. Front. Microbiol. 10 (2019), doi:10.3389/fmicb.2019.00214.

4. D. S. Bower, K. R. Lips, L. Schwarzkopf, A. Georges, S. Clulow, Amphibians on the brink. Science. 357, 454-455 (2017).

5. M. C. Fisher, T. W. J. Garner, Chytrid fungi and global amphibian declines. Nature Reviews Microbiology. 18, 332-343 (2020).

$6 . \quad$ D. S. Blehert, A. C. Hicks, M. Behr, C. U. Meteyer, B. M. Berlowski-Zier, E. L. Buckles, J. T. H. Coleman, S. R. Darling, A. Gargas, R. Niver, J. C. Okoniewski, R. J. Rudd, W. B. Stone, Bat white-nose syndrome: an emerging fungal pathogen? Science. 323, 227-227 (2009).

7. W. F. Frick, J. F. Pollock, A. C. Hicks, K. E. Langwig, D. S. Reynolds, G. G. Turner, C. M. Butchkoski, T. H. Kunz, An emerging disease causes regional population collapse of a common North American bat species. Science. 329, 679-682 (2010).

8. J. M. Lorch, S. Knowles, J. S. Lankton, K. Michell, J. L. Edwards, J. M. Kapfer, R. A. Staffen, E. R. Wild, K. Z. Schmidt, A. E. Ballmann, D. Blodgett, T. M. Farrell, B. M. Glorioso, L. A. Last, S. J. Price, K. L. Schuler, C. E. Smith, J. F. X. Wellehan, D. S. Blehert, Snake fungal disease: an emerging threat to wild snakes. Phil. Trans. R. Soc. B. 371, 20150457 (2016).

9. F. T. Burbrink, J. M. Lorch, K. R. Lips, Host susceptibility to snake fungal disease is highly dispersed across phylogenetic and functional trait space. Science Advances. 3, e1701387 (2017).

10. A. D. Thomas, L. Sigler, S. Peucker, J. H. Norton, A. Nielan, Chrysosporium anamorph of Nannizziopsis vriesii associated with fatal cutaneous mycoses in the salt-water crocodile (Crocodylus porosus). Medical Mycology. 40, 143-151 (2002).

11. J. A. Paré, L. Sigler, An Overview of Reptile Fungal Pathogens in the Genera Nannizziopsis, Paranannizziopsis, and Ophidiomyces. Journal of Herpetological Medicine and Surgery. 26, 46-53 (2016).

12. J. Schneider, T. Heydel, L. Klasen, M. Pees, W. Schrödl, V. Schmidt, Characterization of Nannizziopsis guarroi with genomic and proteomic analysis in three lizard species. Medical Mycology. 56, 610-620 (2018).

13. A. G. Hill, J. R. Sandy, A. Begg, Mycotic dermatitis in juvenile freshwater crocodiles (Crocodylus johnstoni) caused by Nannizziopsis crocodili. zamd. 50, 225-230 (2019).

14. L. Sigler, S. Hambleton, J. A. Paré, Molecular characterization of reptile pathogens currently known as members of the Chrysosporium anamorph of Nannizziopsis vriesii complex and relationship with some human-associated isolates. Journal of Clinical Microbiology. 51, 3338-3357 (2013).

15. N. R. Peterson, K. Rose, S. Shaw, T. H. Hyndman, L. Sigler, D. İ. Kurtböke, J. Llinas, B. L. Littleford-Colquhoun, R. Cristescu, C. Frère, Cross-continental emergence of Nannizziopsis barbatae disease may threaten wild Australian lizards. Sci Rep. 10, 20976 (2020).

16. K. Strickland, R. Gardiner, A. J. Schultz, C. H. Frère, The social life of eastern water dragons: sex differences, spatial overlap and genetic relatedness. Animal Behaviour. 97, 53-61 (2014).

17. R. Z. Gardiner, E. Doran, K. Strickland, L. Carpenter-Bundhoo, C. Frère, A Face in the Crowd: A Non-Invasive and Cost Effective Photo-Identification Methodology to Understand the Fine Scale Movement of Eastern Water Dragons. PLoS ONE. 9, e96992 (2014).

18. J. F. Muñoz, J. G. McEwen, O. K. Clay, C. A. Cuomo, Genome analysis reveals evolutionary mechanisms of adaptation in systemic dimorphic fungi. Scientific Reports. 8, 4473 (2018).

19. I. Yike, Fungal proteases and their pathophysiological effects. Mycopathologia. 171, 299-323 (2011).

20. R. Johnson, C. Sangster, L. Sigler, S. Hambleton, J. Paré, Deep fungal dermatitis caused by the Chrysosporium anamorph of Nannizziopsis vriesii in captive coastal bearded dragons (Pogona barbata). Australian Veterinary Journal. 89, 515-519 (2011). 
21. R. A. Farrer, A. Martel, E. Verbrugghe, A. Abouelleil, R. Ducatelle, J. E. Longcore, T. Y. James, F. Pasmans, M. C. Fisher, C. A. Cuomo, Genomic innovations linked to infection strategies across emerging pathogenic chytrid fungi. Nature Communications. 8, 14742 (2017).

22. K. A. Field, J. S. Johnson, T. M. Lilley, S. M. Reeder, E. J. Rogers, M. J. Behr, D. M. Reeder, The White-Nose Syndrome Transcriptome: Activation of Anti-fungal Host Responses in Wing Tissue of Hibernating Little Brown Myotis. PLOS Pathogens. 11, e1005168 (2015).

23. E. L. Pannkuk, T. S. Risch, B. J. Savary, Isolation and Identification of an Extracellular SubtilisinLike Serine Protease Secreted by the Bat Pathogen Pseudogymnoascus destructans. PLOS ONE. 10, e0120508 (2015).

24. C. M. Davy, M. E. Donaldson, H. Bandouchova, A. M. Breit, N. A. S. Dorville, Y. A. Dzal, V. Kovacova, E. L. Kunkel, N. Martínková, K. J. O. Norquay, J. E. Paterson, J. Zukal, J. Pikula, C. K. R. Willis, C. J. Kyle, Transcriptional host-pathogen responses of Pseudogymnoascus destructans and three species of bats with white-nose syndrome. Virulence. 11, 781-794 (2020).

25. Y.-S. Bahn, C. Xue, A. Idnurm, J. C. Rutherford, J. Heitman, M. E. Cardenas, Sensing the environment: lessons from fungi. Nature Reviews Microbiology. 5, 57-69 (2007).

26. D. A. Martinez, B. G. Oliver, Y. Gräser, J. M. Goldberg, W. Li, N. M. Martinez-Rossi, M. Monod, E. Shelest, R. C. Barton, E. Birch, A. A. Brakhage, Z. Chen, S. J. Gurr, D. Heiman, J. Heitman, I. Kosti, A. Rossi, S. Saif, M. Samalova, C. W. Saunders, T. Shea, R. C. Summerbell, J. Xu, S. Young, Q. Zeng, B. W. Birren, C. A. Cuomo, T. C. White, Comparative genome analysis of trichophyton rubrum and related dermatophytes reveals candidate genes involved in infection. mBio. 3 (2012), doi:10.1128/mBio.00259-12. 27. R. de Jonge, H. P. van Esse, A. Kombrink, T. Shinya, Y. Desaki, R. Bours, S. van der Krol, N. Shibuya, M. H. A. J. Joosten, B. P. H. J. Thomma, Conserved fungal lysM effector Ecp6 prevents chitintriggered immunity in plants. Science. 329, 953-955 (2010).

28. J. Antonovics, Transmission dynamics: critical questions and challenges. Philosophical Transactions of the Royal Society B: Biological Sciences. 372, 20160087 (2017).

29. S. Altizer, A. Dobson, P. Hosseini, P. Hudson, M. Pascual, P. Rohani, Seasonality and the dynamics of infectious diseases. Ecol Lett. 9, 467-484 (2006).

30. G. F. Albery, L. Kirkpatrick, J. A. Firth, S. Bansal, Unifying spatial and social network analysis in disease ecology. J Anim Ecol. 90, 45-61 (2021).

31. C. J. Briggs, R. A. Knapp, V. T. Vredenburg, Enzootic and epizootic dynamics of the chytrid fungal pathogen of amphibians. PNAS. 107, 9695-9700 (2010).

32. C. A. Dobony, A. C. Hicks, K. E. Langwig, R. I. von Linden, J. C. Okoniewski, R. E. Rainbolt, Little Brown Myotis Persist Despite Exposure to White-Nose Syndrome. Journal of Fish and Wildlife Management. 2, 190-195 (2011).

33. J. M. McKenzie, S. J. Price, G. M. Connette, S. J. Bonner, J. M. Lorch, Effects of snake fungal disease on short-term survival, behavior, and movement in free-ranging snakes. Ecological Applications. 31, e02251 (2021).

34. K. E. Langwig, W. F. Frick, J. T. Bried, A. C. Hicks, T. H. Kunz, A. M. Kilpatrick, Sociality, density-dependence and microclimates determine the persistence of populations suffering from a novel fungal disease, white-nose syndrome. Ecology Letters. 15, 1050-1057 (2012).

35. B. C. Scheele, L. F. Skerratt, L. F. Grogan, D. A. Hunter, N. Clemann, M. McFadden, D. Newell, C. J. Hoskin, G. R. Gillespie, G. W. Heard, L. Brannelly, A. A. Roberts, L. Berger, After the epidemic: Ongoing declines, stabilizations and recoveries in amphibians afflicted by chytridiomycosis. Biological Conservation. 206, 37-46 (2017).

36. S. Benhaiem, L. Marescot, M. L. East, S. Kramer-Schadt, O. Gimenez, J.-D. Lebreton, H. Hofer, Slow recovery from a disease epidemic in the spotted hyena, a keystone social carnivore. Commun Biol. 1, 201 (2018).

37. K. Wells, R. K. Hamede, D. H. Kerlin, A. Storfer, P. A. Hohenlohe, M. E. Jones, H. I. McCallum, Infection of the fittest: devil facial tumour disease has greatest effect on individuals with highest reproductive output. Ecol Lett. 20, 770-778 (2017).

38. C. M. Lind, J. M. Lorch, I. T. Moore, B. J. Vernasco, T. M. Farrell, Seasonal sex steroids indicate reproductive costs associated with snake fungal disease. Journal of Zoology. 307, 104-110 (2019). 
401

402

403

404

405

406

407

408

409

410

411

412

413

414

415

416

417

418

419

420

421

422

423

424

425

426

427

428

429

430

431

432

433

434

435

436

437

438

439

440

441

442

443

444

445

446

39. C. Kindermann, E. J. Narayan, J.-M. Hero, Does physiological response to disease incur cost to reproductive ecology in a sexually dichromatic amphibian species? Comparative Biochemistry and Physiology Part A: Molecular \& Integrative Physiology. 203, 220-226 (2017).

40. H. McCallum, N. Barlow, J. Hone, How should pathogen transmission be modelled? Trends in Ecology \& Evolution. 16, 295-300 (2001).

41. A. Gargas, M. T. Trest, M. Christensen, T. J. Volk, D. S. Blehert, <I $>$ Geomyces destructans $</ \mathrm{I}>$ sp. nov. associated with bat white-nose syndrome. Mycotaxon. 108, 147-154 (2009).

42. L. Berger, R. Speare, P. Daszak, D. E. Green, A. A. Cunningham, C. L. Goggin, R. Slocombe, M. A. Ragan, A. D. Hyatt, K. R. McDonald, H. B. Hines, K. R. Lips, G. Marantelli, H. Parkes, Chytridiomycosis causes amphibian mortality associated with population declines in the rain forests of Australia and Central America. PNAS. 95, 9031-9036 (1998).

43. M. B. Thompson, Estimate of the population structure of the estern water dragon, Physignathus lesueurii (Reptilia : Agamidae), along riverside habitat. Wildl. Res. 20, 613-619 (1993).

44. V. Ter-Hovhannisyan, A. Lomsadze, Y. O. Chernoff, M. Borodovsky, Gene prediction in novel fungal genomes using an ab initio algorithm with unsupervised training. Genome Res. 18, 1979-1990 (2008).

45. M. Stanke, O. Schöffmann, B. Morgenstern, S. Waack, Gene prediction in eukaryotes with a generalized hidden Markov model that uses hints from external sources. BMC Bioinformatics. 7, 62 (2006).

46. I. Korf, Gene finding in novel genomes. BMC Bioinformatics. 5, 59 (2004).

47. A. L. Delcher, K. A. Bratke, E. C. Powers, S. L. Salzberg, Identifying bacterial genes and endosymbiont DNA with Glimmer. Bioinformatics. 23, 673-679 (2007).

48. B. J. Haas, S. L. Salzberg, W. Zhu, M. Pertea, J. E. Allen, J. Orvis, O. White, C. R. Buell, J. R. Wortman, Automated eukaryotic gene structure annotation using EVidenceModeler and the Program to Assemble Spliced Alignments. Genome Biol. 9, R7 (2008).

49. F. A. Simão, R. M. Waterhouse, P. Ioannidis, E. V. Kriventseva, E. M. Zdobnov, BUSCO: assessing genome assembly and annotation completeness with single-copy orthologs. Bioinformatics. 31, 3210-3212 (2015).

50. R. D. Finn, A. Bateman, J. Clements, P. Coggill, R. Y. Eberhardt, S. R. Eddy, A. Heger, K. Hetherington, L. Holm, J. Mistry, E. L. L. Sonnhammer, J. Tate, M. Punta, Pfam: the protein families database. Nucleic Acids Research. 42, D222-D230 (2014).

51. S. C. Potter, A. Luciani, S. R. Eddy, Y. Park, R. Lopez, R. D. Finn, HMMER web server: 2018 update. Nucleic Acids Research. 46, W200-W204 (2018).

52. R. C. Team, R: A Language and Environment for Statistical Computing (R Foundation for Statistical Computing, Vienna, Austria., 2021).

53. R. C. Edgar, MUSCLE: a multiple sequence alignment method with reduced time and space complexity. BMC Bioinformatics. 5, 113 (2004).

54. M. N. Price, P. S. Dehal, A. P. Arkin, FastTree 2 - Approximately Maximum-Likelihood Trees for Large Alignments. PLOS ONE. 5, e9490 (2010).

55. S. Martino, H. Rue, Implementing Approximate Bayesian Inference using Integrated Nested Laplace Approximation: a manual for the inla program. Department of Mathematical Sciences, NTNU, Norway. (2009).

56. F. Lindgren, H. Rue, J. Lindström, An explicit link between Gaussian fields and Gaussian Markov random fields: the stochastic partial differential equation approach. Journal of the Royal Statistical Society: Series B (Statistical Methodology). 73, 423-498 (2011).

57. F. Lindgren, H. Rue, Bayesian Spatial Modelling with R-INLA. Journal of Statistical Software. 63, $1-25$ (2015). 
Table 1. Details of the fungal species used in the comparative analysis.

\begin{tabular}{|c|c|c|c|c|c|c|c|c|c|}
\hline Species & Division & Order & Family & Host & Disease & $\begin{array}{l}\text { GenBank } \\
\text { Accession }\end{array}$ & $\begin{array}{l}\text { Genome } \\
\text { Size } \\
(\mathrm{MB})\end{array}$ & $\begin{array}{l}\text { Genome } \\
\mathrm{N} 50 \\
(\mathrm{~Kb})\end{array}$ & $\begin{array}{l}\text { Number } \\
\text { predicted } \\
\text { proteins }\end{array}$ \\
\hline Nannizziopsis barbatae & Ascomycota & Onygenales & Onygenaceae & Reptiles & Dermatomycoses & GCA_014964245.1 & 31.543 & 6,192 & $8,012 *$ \\
\hline Ophidiomyces ophiodiicola & Ascomycota & Onygenales & Onygenaceae & Snakes & Ophidiomycosis (snake fungal disease) & GCA_002167195.1 & 21.865 & 1,499 & $6,983^{*}$ \\
\hline Uncinocarpus reesii & Ascomycota & Onygenales & Onygenaceae & - & Non-pathogenic & GCF_000003515.1 & 22.349 & 5,232 & 7,760 \\
\hline Coccidioides immitis & Ascomycota & Onygenales & Onygenaceae & Humans & Coccidioidomycosis (valley fever) & GCA_004115165.2 & 27.474 & 3,797 & 7,815 \\
\hline Chrysosporium queenslandicum & Ascomycota & Onygenales & Onygenaceae & - & Non-pathogenic & GCA_001430955.1 & 32.335 & 173 & $11564 *$ \\
\hline Microsporum canis & Ascomycota & Onygenales & Arthrodermataceae & Humans, animals & Dermatophytosis & GCF_000151145.1 & 23.263 & 2,919 & 8,765 \\
\hline Trichophyton rubrum & Ascomycota & Onygenales & Arthrodermataceae & Humans & Dermatophytosis & GCF_000151425.1 & 22.530 & 2,156 & 8,706 \\
\hline Trichophyton equinum & Ascomycota & Onygenales & Arthrodermataceae & Humans, horses & Dermatophytosis & GCA_000151175.1 & 24.158 & 397 & 8,676 \\
\hline Nannizzia gypsea & Ascomycota & Onygenales & Arthrodermataceae & Humans, animals & Dermatophytosis, onychomycosis & GCF_000150975.2 & 23.272 & 3,227 & 8,921 \\
\hline Paracoccidioides brasiliensis & Ascomycota & Onygenales & Ajellomycetaceae & Humans & Paracoccidioidomycosis & GCF_000150735.1 & 29.952 & 2,149 & 8,390 \\
\hline Blastomyces dermatitidis & Ascomycota & Onygenales & Ajellomycetaceae & Humans, animals & Blastomycosis & GCA_000151595.1 & 73.633 & 400 & 11,443 \\
\hline Pseudogymnoascus destructans & Ascomycota & Incertae sedis & Pseudeurotiaceae & Bats & White-nose syndrome & GCF_001641265.1 & 35.818 & 1,168 & 9,405 \\
\hline Saccharomyces cerevisiae & Ascomycota & Saccharomycetales & Saccharomycetaceae & - & Non-pathogenic & GCF_000146045.2 & 12.157 & 924 & 6,002 \\
\hline Candida albicans & Ascomycota & Saccharomycetales & Saccharomycetaceae & Humans & Candidiasis & GCF_000182965.3 & 14.282 & 2,231 & 6,030 \\
\hline Aspergillus fumigatus & Ascomycota & Eurotiales & Trichocomaceae & Humans & Aspergillosis & GCA_000002655.1 & 29.384 & 3,948 & 9,630 \\
\hline Cryptococcus neoformans & Basidiomycota & Tremellales & Tremellaceae & Humans & Cryptococcosis & GCF_000091045.1 & 19.051 & 4,438 & 6,863 \\
\hline Batrachochytrium dendrobatidis & Chytridiomycota & Rhizophydiales & Batrachochytriaceae & Amphibians & Chytridiomycosis & GCF_000203795.1 & 24.315 & 1,484 & 8,677 \\
\hline
\end{tabular}

* Gene annotations produced in this study. 
bioRxiv preprint doi: https://doi.org/10.1101/2021.10.22.465237; this version posted October 24, 2021. The copyright holder for this preprint (which was not certified by peer review) is the author/funder, who has granted bioRxiv a license to display the preprint in perpetuity. It is made available under aCC-BY 4.0 International license.
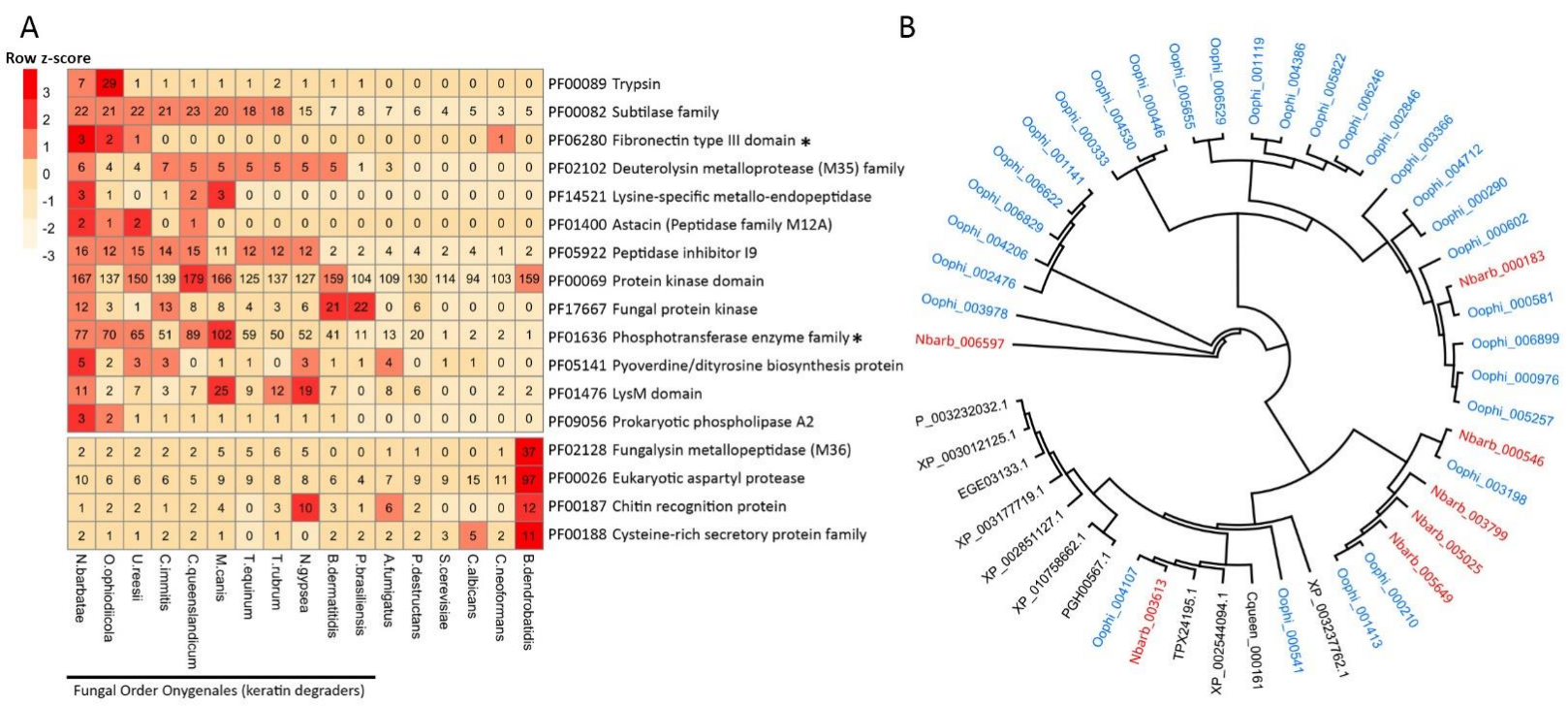

Fig. 1. Comparative genomic analysis of $N$. barbatae with other fungal species. (A) Gene family size comparison of putative proteases and other proteins implicated in fungal virulence. Protein families significantly expanded in $N$. barbatae are marked with *. (B) Phylogenetic relationship of the trypsin domain-containing protein sequences identified in this study, red text, $N$. barbatae proteins; blue text, $O$. ophidiicola proteins. 


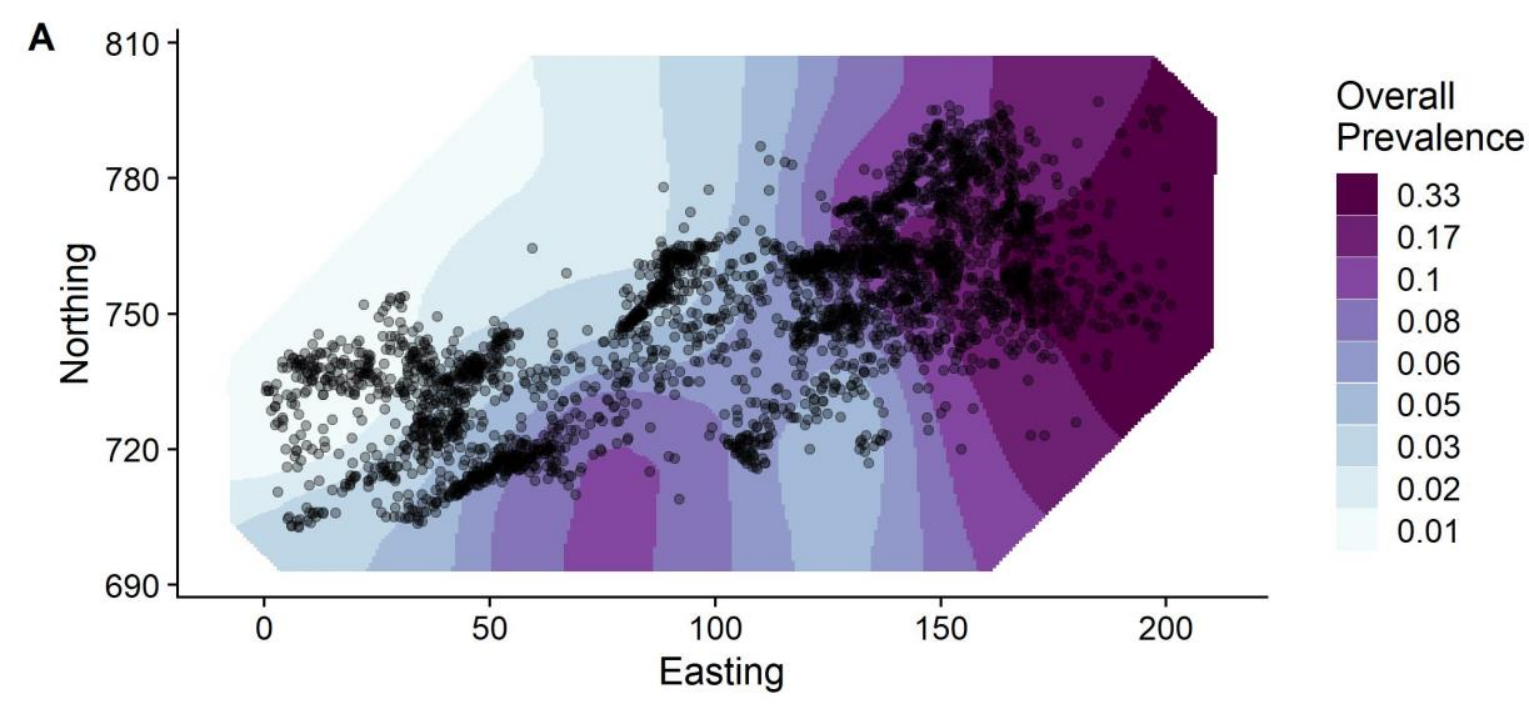

B
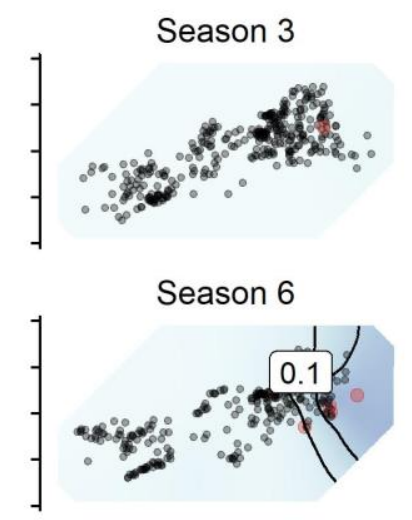

Season 9

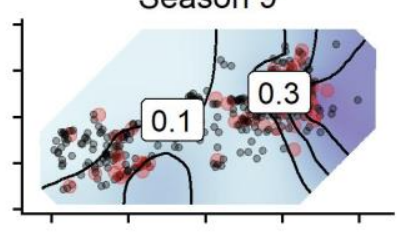

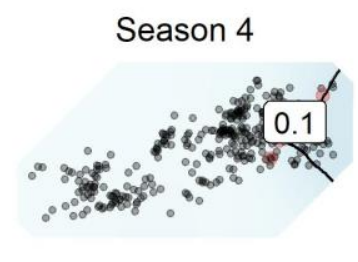

Season 7

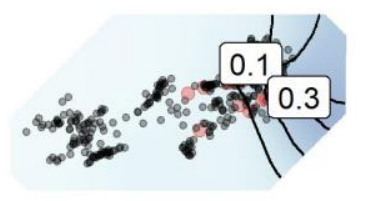

Season 10

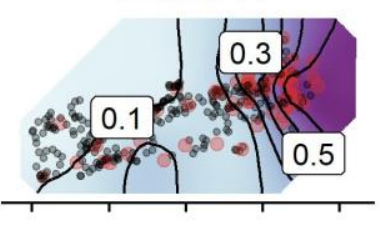

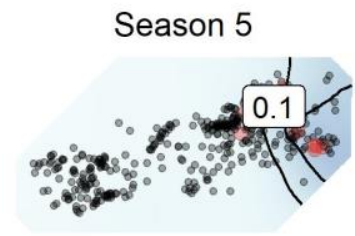

Season 8

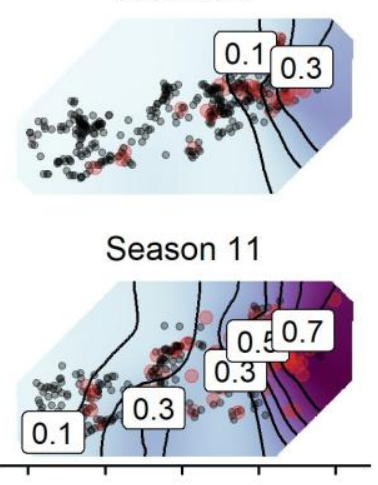

Fungus

- 0

- 1

Annual Prevalence

0.6

0.4

0.2

\section{Fig. 2. Spatial distribution and temporal spread of $N$. barbatae infection within the}

population. These are represented as the spatial distribution of the spatial random effect from the Spatial model (A) and the annually stratified Spatiotemporal model (B), respectively. The spatial effects were estimated using a stochastic partial differentiation equation (SPDE) in an integrated nested Laplace approximation (INLA) model. Adding these SPDE components substantially 
improved model fit. In (A), points represent individuals' average annual locations. The axes in (B) are identical to those in (A), with the labels removed for plotting clarity. In (A), the spatial effect is categorized into eight quantiles to facilitate visualization over a range of prevalence values. 

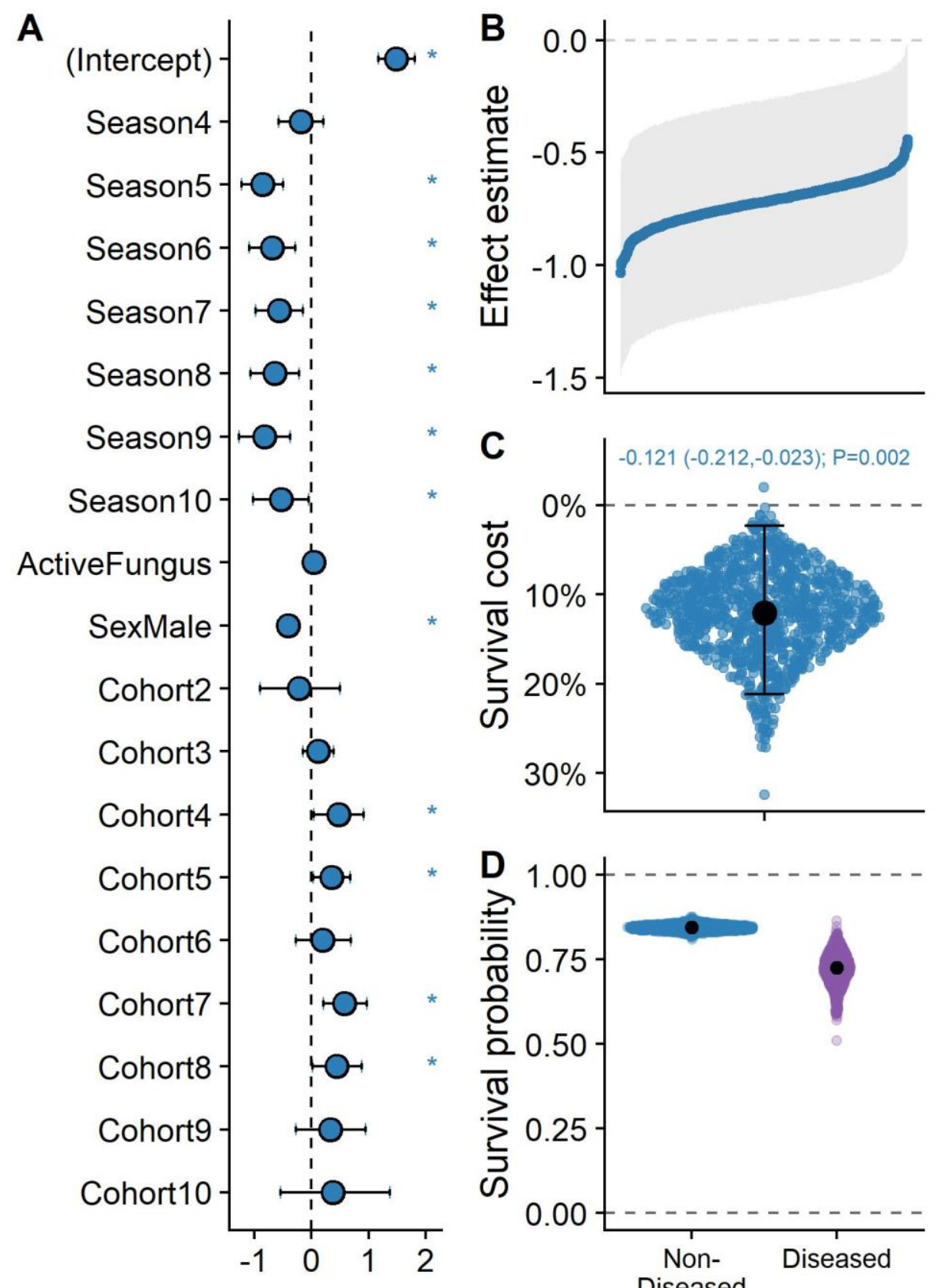

Estimate

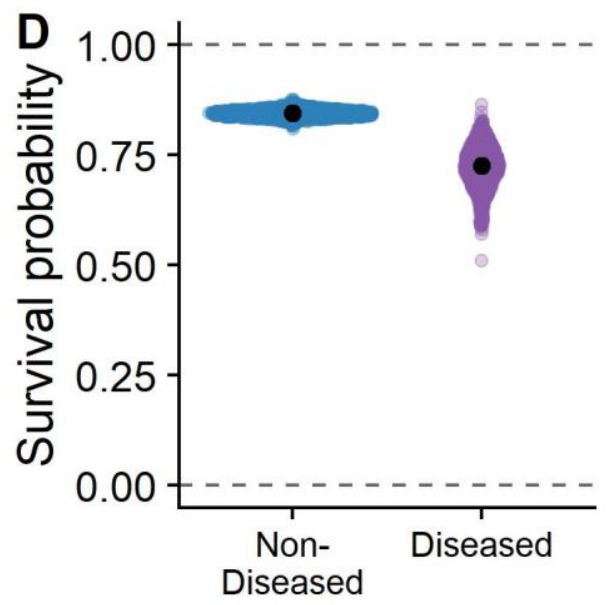

Fig.3. Survival effects of $N$. barbatae infection in the population. Survival effects of the disease are estimated using a full-population model (A), and an approach combining model uncertainty and subsampling regime uncertainty $(\mathbf{B}, \mathbf{C}, \mathbf{D})$. The second approach provided estimates of survival effects across all subsamples (B) and survival costs (C) and probabilities (D) of diseased 
vs. non-diseased individuals. The large black points represent means across all 1000 replicates.

The text at the top of panel C displays the effect estimate for the survival cost across all models, with $95 \%$ credibility intervals in brackets and the $\mathrm{P}$ value. The error bars represent the $95 \%$ credibility intervals. 